\title{
Percepción y nivel de comprensión del consentimiento informado en la cirugía del tercer molar inferior

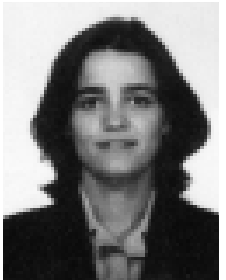 \\ López-Carriches, Carmen
}

\section{Perception and level of understanding of the informed consent in lower third molar surgery}

López-Carriches, Carmen*

Baca Pérez-Bryan, Rafael**

San Hipólito-Marín, Lara***

Donado-Rodríguez, Manuel****

*Profesora colaboradora de Cirugía de la Facultad de Odontología (UCM) y asociada de la UEM.

**Profesor titular de Cirugía. UCM. ***Magíster en Cirugía Bucal por la UCM.

****Catedrático de Patología quirúrgica Oral y Maxilofacial. UCM.

\begin{abstract}
Resumen: La firma del consentimiento informado por parte del paciente es obligatoria antes de cualquier intervención. Objetivos: Los objetivos de este artículo original fueron: -Conocer el nivel de comprensión del consentimiento informado por parte del paciente. -Determinar el porcentaje de pacientes que rechazan el tratamiento al conocer los riesgos del mismo. Pacientes y metodo: Participaron en el estudio 385 pacientes a los que se les extrajo el tercer molar quirúrgicamente tras haber firmado el consentimiento informado. Posteriormente se les realizó una encuesta para comprobar si habían comprendido la finalidad del consentimiento informado y su opinión sobre el procedimiento. Resultados: Los datos se trataron mediante estadística descriptiva. L más destacable es que el $100 \%$ de los pacientes firmaron el consentimiento informado y se sometieron a la cirugía. El 99,74\%entendió claramente qué le harían en la intervención. El $97 \%$ sabía que se podían presentar complicaciones y el $94 \%$ entendió que podía negarse a la intervención. Más de la mitad $(61,56 \%)$ pensaba que el consentimiento sirve para eximir de responsabilidad al profesional. Al 98,96\%les parece útil este tipo de información. Conclusiones: El consentimiento informado fue comprendido por la mayoría de los pacientes y ninguno de ellos se negó a la intervención.
\end{abstract}

Palabras clave: Consentimiento informado, Cirugía, Cirugía Bucal.

Abstract: The signing of an informed consent by the patient is required before any operation. Objetives: The objectives of this original article were. -To know the level of understanding of the informed consent by the patient. -To determine the percentage of patients who avoid the treatment when knowing the risks of it. Patients and method: 385 patients, who were sudmitted to surgical third molar extraction after signing the informed consent. After that, a survey was carried out to check if they had understood the aim of informed consent as well as their opinion about this procedure. Results: Data were processed with descriptive statistics. The most remarkable thing is that $100 \%$ of the patients signed the informed consent and they agreed on the surgery. $99.74 \%$ understood clearly what was going to be done during the operation. $97 \%$ knew that complications might occur and $94 \%$ were aware of their possibility of denying the operation. More than half of them (61.56\%) thought that informed consent serves for exempting the professional from responsibility. This kind of information is thought to be useful for $98.96 \%$ Conclusions: The informed consent was understood by the majority of the patients and none of them avoided the operation.

Key words: Informed consent, Surgery, Oral Surgery.

\begin{tabular}{ccc}
\hline $\begin{array}{c}\text { Fecha recepción } \\
\text { 31-1-2003 }\end{array}$ & Fecha última revisión & $\begin{array}{c}\text { Fecha aceptación } \\
30-10-2003\end{array}$ \\
\hline
\end{tabular}

BIBUD [1138-123X (2003)8:6; noviembre-diciembre 593-724]

López-Carriche C, Baca Pérez-Bryan R, San Hipólito-Marín L, Donado-Rodríguez M. Percepción y nivel de comprensión del consentimiento informado en la cirugía del tercer molar inferior. RCOE 2003;8(6):633-641.
Carmen López Carriches

C/. Rey Francisco, 11. Bajo Izda.

28008 - Madrid 


\section{Introducción}

Las demandas contra dentistas están aumentando de forma lenta pero constante en los últimos años ${ }^{1 * *}$. La mejor protección contra la denuncia por «mal praxis» es la historia clínica y el consentimiento informado.

En Madrid el mayor número de demandas se produce en los tratamientos de prótesis fija. En segundo lugar están las reclamaciones por endodoncias, la prótesis parcial removible y los tratamientos de odontología conservadora. En menor proporción se denuncian casos de ortodoncia, cirugía, implantes, periodoncia, prótesis completa (no en vano cada vez se hacen menos prótesis completas) y anestesia y exodoncia simple ${ }^{2 * *}$.

Como podemos observar la cirugía bucal no es de las especialidades que más demandas recibe; sin embargo, cuando éstas se producen pueden acabar con facilidad en una condena para el dentista ${ }^{3}$.

El principal problema que plantea la extracción quirúrgica del tercer molar inferior es que siempre aparecen, en mayor o menor cuantía, una triada sintomática constituida por inflamación, dolor y trismo 4 (fig. 1). Con menor frecuencia, la cirugía del tercer molar inferior puede venir seguida de complicaciones de tipo infeccioso, lesiones de los nervios dentario o lingual, daño de dientes adyacentes, fracturas óseas, etc. (fig. 2).

Son muchos los estudios que se han realizado sobre las complicaciones de la cirugía del tercer molar inferior. Conrad y cols $^{5 * *}$ en un estudio realizado en 1999 llegaron a la conclusión de que el peor postoperatorio se

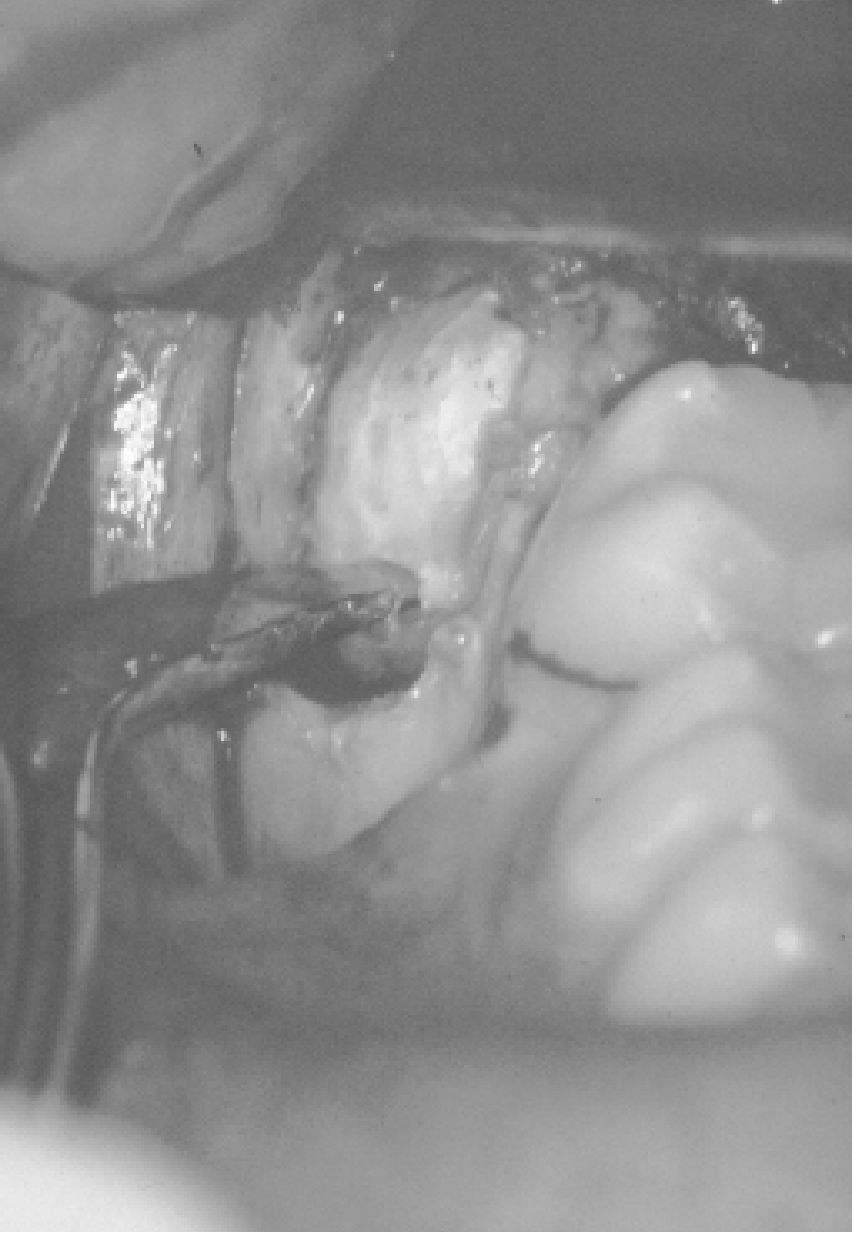

Figura 1. Cirugía del tercer molar inferior.

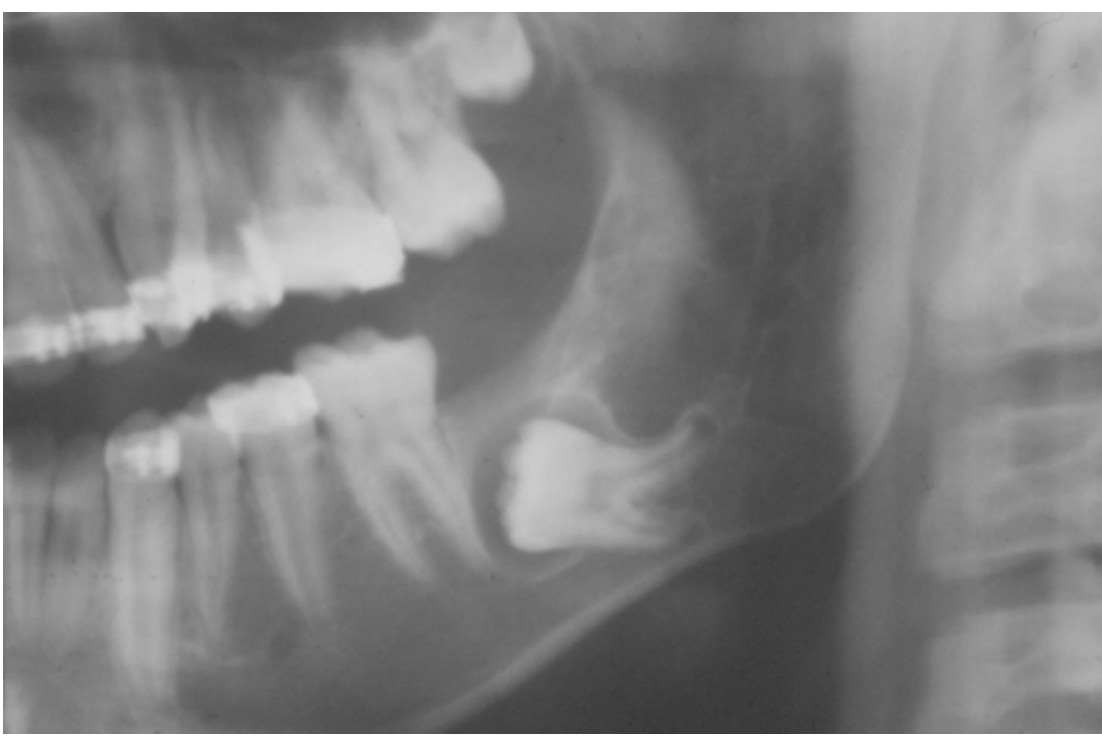

Figura 2. Tercer molar inferior en relación con el nervio dentario.

RCOE, 2003, Vol 8, №6, 633-641 
daba cuando la cirugía se prolongaba en el tiempo, cuando los terceros molares estaban por debajo del plano oclusal y en mujeres. Esto corrobora, como todos sabemos, que los riesgos son reales y que los cirujanos deben incluirlos en los consentimientos informados, tanto de forma oral como de forma escrita.

Para evitar posibles reclamaciones por «mal praxis» es importante asegurar una adecuada comprensión de los riesgos, beneficios y alternativas de la extracción del tercer molar. Para que el consentimiento sea adecuado, los pacientes deben ser capaces de comprender los cuatro componentes del consentimiento informado: razones del tratamiento, riesgos, beneficios y alternativas. Es decir, no basta con obtener la firma del paciente, debemos estar seguros de que el paciente ha comprendido la información.

En algunas investigaciones se ha comprobado que los pacientes no comprenden adecuadamente los componentes del consentimiento informado ${ }^{6 * *}$. A los pacientes se les debe dar una información de acuerdo a su nivel de comprensión. Se les debe ayudar a comprender en qué situación está su tercer molar, las razones por las que la extracción ayudará o perjudicará al paciente y las posibles complicaciones si el paciente decide no extraer su cordal. Esta información debe ser presentada de forma escrita, verbal y en ocasiones gráfica, para asegurarnos de que el paciente comprende su contenido.

Cannavina $^{7 * *}$ cita algunos problemas relacionados con la obtención del consentimiento informado, como son:

- Cuánta información dar al paciente en una situación específica.
- Qué capacidad tiene el paciente para comprender la información dada.

- Cuál es el nivel de comprensión alcanzado por el paciente.

La libertad del clínico ha sido reducida y ahora es necesario realizar un consentimiento informado que «no es válido a menos que el paciente comprenda la naturaleza y propósito del tratamiento». Una vez que una persona es informada ampliamente del procedimiento y da su consentimiento éste es válido; es decir, el profesional debe dar al paciente una completa información sobre los benéficos y los riesgos.

Por todo lo anterior nos planteamos realizar el presente trabajo cuyos objetivos son:

- Conocer el nivel de comprensión del consentimiento informado por parte del paciente.

- Determinar el porcentaje de pacientes que rechazan el tratamiento al conocer los riesgos del mismo.

\section{Pacientes y método}

Participaron en el estudio 385 pacientes que acudieron a la Unidad de Cirugía Bucal (Departamento de Medicina y Cirugía Bucofacial) de la Facultad de Odontología de la Universidad Complutense de Madrid para la extracción quirúrgica de terceros molares. El estudio tuvo una duración de tres meses y medio.

Los criterios de inclusión fueron los siguientes: pacientes que precisaban extracción quirúrgica de terceros molares parcial o completamente retenidos, que daban su consentimiento para participar en el estudio, mayores de 18 años.
En cuanto a la potencia del estudio basándonos en el tamaño de la muestra, para proporciones esperadas mayores o iguales a 0,2 , el tamaño $n=385$ proporciona errores inferiores al 3\% En el caso más desfavorable, proporciones esperadas iguales a 0,5 y $\mathrm{n}=385$ proporciona estimaciones con un error del $5 \%( \pm 0,05)$. Por lo tanto, es un tamaño de muestra suficiente, que se limitó por razones de duración del estudio.

Antes de la cirugía se le entregó a cada paciente el consentimiento informado por escrito (Anexo 1) y se le explicó también verbalmente las particularidades de la intervención quirúrgica. Se le permitió hacer todo tipo de preguntas para subsanar posibles dudas y lo firmó. También se le pidió que cuantificará su grado de ansiedad ante la intervención de 0 a 10, considerando de 0 a 3 ansiedad baja, de 4 a 6 media y de 7 a 10 alta. Una vez concluida la extracción del tercer molar se le realizó una pequeña encuesta con los datos de filiación, su ocupación y su nivel de estudios (sin estudios o estudios primarios, con estudios secundarios o universitarios) y se le hicieron diez preguntas concretas sobre el consentimiento informado que acababa de firmar:

1. ¿Le han explicado claramente qué operación le iban a hacer?

2. ¿Entendió claramente qué le harían en la operación?

3. ¿Le explicaron si la operación a la que se sometería podía presentar algún peligro o alguna complicación?

4. ¿Pudo preguntar todo lo que quiso sobre su intervención y sus posibles consecuencias al cirujano?

5. ¿Sabía que podía negarse a la operación? 


\section{Anexo 1 Formulario de consentimiento informado para la extracción de cordales}

FECHA:

NOMBRE Y APEWDOS DEL PACIENTE:

NOMBRE DEL CIRUJ ANO QUE INFORMA:

GRADO DE ANSIEDAD DEL PACIENTE (DE 0 A 10):

Se va a proceder a la extracción del molar por el siguiente motivo

El procedimiento se llevará a cabo bajo anestesia local (con los riesgos inherentes a la misma). Es una intervención de cirugía bucal que no está exenta de complicaciones, tales como:

- Alergia al anestésico u otro medicamento utilizado, antes, durante o después de la cirugía.

- Inflamación (que se controlará con fármacos).

- Dolor (que se controlará con fármacos).

- Trismo o disminución transitoria de la apertura bucal.

- Hematoma.

- Dehiscencia de la sutura.

- Daño de dientes adyacentes.

- Hipoestesia o anestesia del nervio dentario inferior, que en la mayoría de los casos es temporal y en menor porcentaje definitiva. (Sólo en la extracción del tercer molar inferior).

- Hipoestesia o anestesia del nervio lingual, temporal o definitiva. (Sólo en la extracción del tercer molar inferior)

- Infección postoperatoria (que se suele controlar farmacológicamente).

- Osteitis.

- Sinusitis. (Sólo en terceros molares superiores).

- Osteomielitis.

- Comunicación orosinusal. (Sólo en terceros molares superiores).

- Fracturas óseas.

- Rotura de instrumentos.

Recibida la anterior información, considero que he comprendido la naturaleza y propósito del procedimiento. Además, en entrevista personal con el Facultativo/a he sido informado/a, en términos asequibles, del alcance de dicho tratamiento. En la entrevista he tenido oportunidad de proponer y resolver mis posibles dudas, y de obtener cuanta información he creido necesaria.

DOY MI CONSENTIMIENTO para que se me practique la extracción quirúrgica del tercer molar ( $n$ o.....)

Este consentimiento puede ser revocado por mí sin necesidad de justificación alguna, en cualquier momento antes de realizar el procedimiento.

Y para que conste, firmo el presente original después de leído.

En Madrid, a. de. de 20

Firma del paciente y DNI

Anexo: Preguntas realizadas por el paciente.
Firma del facultativo

№ de colegiado

RCOE, 2003, Vol 8, №6, 633-641 
6. ¿Piensa usted que el consentimiento exime de responsabilidad al personal que le opera?

7. ¿Podía enumerarme alguna de las complicaciones que le han explicado?

8. ¿Le parece útil este tipo de información?

9. ¿El conocer las posibles complicaciones ha aumentado en usted el nivel de ansiedad?

10. ¿El conocer las complicaciones le tranquiliza?

Los datos recogidos en estas encuestas se trataron mediante estadística descriptiva, usando para tal fin el programa estadístico BMDP. A continuación describimos, muy brevemente, los programas utilizados para cada test estadístico concreto.

-BMDP2D. Descripción detallada de datos. Frecuencias. Calcula frecuencias y porcentajes de cada valor particular. Calcula media, mediana y moda, errores estándar de la media y mediana, etc.

-BMDP4F. Tablas de frecuencias. Analiza datos categóricos o cualitativos en tablas de frecuencias.

\section{Resultados}

Se extrajeron un total de 385 cordales: 38 superiores derechos, 36 superiores izquierdos, 155 inferiores derechos y 156 inferiores izquierdos.

En cuanto a los aspectos epidemiológicos, la edad media de los pacientes fue de 25,84 años con un rango comprendido entre los 18 y los 72 años. 234 pacientes eran mujeres $(60,78 \%$ y 151 eran varones $(39,22 \%$.

El $49,35 \%$ de los pacientes tenía estudios secundarios, el $35,06 \%$ no

\begin{tabular}{|lcc|}
\hline $\begin{array}{l}\text { Tabla li: Contestación a los pacientes a la pregunta ile explicaron si la } \\
\text { operación a la que se sometia podía presentar algún peligro o alguna } \\
\text { complicación? Según el sexo, nivel de estudios y nivel de ansiedad }\end{array}$ \\
\hline \multicolumn{1}{|c|}{ SI } & No \\
\hline MUJ ERES & $229(97,86 \%)$ & $5(2,14 \%)$ \\
\hline HOMBRES & $146(96,69 \%)$ & $5(3,31 \%)$ \\
\hline SIN ESTUDIOS O PRIMARIOS & $59(98,33 \%)$ & $1(1,67 \%)$ \\
\hline ESTUDIOS SECUNDARIOS & $183(96,32 \%)$ & $7(3,68 \%)$ \\
\hline ESTUDIOS UNIVERSITARIOS & $133(98,52 \%)$ & $2(1,48 \%)$ \\
\hline ANSIEDAD BAJ A & $117(96,69 \%)$ & $4(3,31 \%)$ \\
\hline ANSIEDAD MEDIA & $146(97,33 \%)$ & $4(2,67 \%)$ \\
\hline ANSIEDAD ALTA & $112(98,25 \%)$ & $2(1,75 \%)$ \\
\hline
\end{tabular}

\begin{tabular}{|lcc|}
\hline \multicolumn{3}{|c|}{$\begin{array}{c}\text { Tabla 2: Contestación a la pregunta isabía que podia negarse a la } \\
\text { operación? Según el sexo, nivel de estudios y nivel de ansiedad }\end{array}$} \\
\hline & $\mathbf{S I}$ & NO \\
\hline MUJ ERES & $220(94,02 \%)$ & $14(5,98 \%)$ \\
\hline HOMBRES & $140(92,72 \%)$ & $11(7,28 \%)$ \\
\hline SIN ESTUDIOS O PRIMARIOS & $58(96,67 \%)$ & $2(3,33 \%)$ \\
\hline ESTUDIOS SECUNDARIOS & $174(91,58 \%)$ & $16(8,42 \%)$ \\
\hline ESTUDIOS UNIVERSITARIOS & $128(94,81 \%)$ & $7(5,19 \%)$ \\
\hline ANSIEDAD BAJ A & $113(93,39 \%)$ & $8(6,61 \%)$ \\
\hline ANSIEDAD MEDIA & $138(92,00 \%)$ & $12(8,00 \%)$ \\
\hline ANSIEDAD ALTA & $109(95,61 \%)$ & $5(4,39 \%)$ \\
\hline
\end{tabular}

tenía estudios o sólo primarios y el 15,58\%tenía estudios universitarios.

El grado de ansiedad ante la cirugía era bajo en un $31,43 \%$ de los casos, medio en un $38,96 \%$ y alto en el $29,61 \%$ de las ocasiones.

En cuanto a la encuesta sobre el consentimiento informado, es de destacar que el $100 \%$ de los pacientes firmaron el consentimiento informado y se sometieron a la cirugía.

La primera pregunta «ile han expli- cado claramente qué operación le iban a hacer?» fue contestada afirmativamente por 384 pacientes y sólo uno negaba haber recibido información sobre la operación. Este mismo paciente contestó también negativamente a la segunda pregunta: «ientendió claramente qué le harían en la operación?», afirmando haberlo entendido el 99,74\% restante. Este paciente era varón, con estudios secundarios y grado de ansiedad bajo. 
A la tercera pregunta: «ile explicaron si la operación a la que se sometería podía presentar algún peligro o alguna complicación?» contestaron afirmativamente un $97 \%$ No hubo diferencias significativas en la contestación entre hombres, mujeres, nivel de estudios o grado de ansiedad (tabla 1).

El $100 \%$ de los pacientes respondieron que sí pudieron preguntar todo lo que quisieron sobre su intervención y sus posibles consecuencias.

En cuanto a la quinta pregunta « ¿sabía que podía negarse a la intervención?» el 93,37\% de los pacientes sabían que podían no someterse a la cirugía del tercer molar al ser informados, no habiendo diferencias significativas en cuanto al sexo, nivel de estudios o grado de ansiedad, como puede apreciarse en la tabla 2.

Al ser preguntados sobre si piensan que el consentimiento exime de responsabilidad al personal que le opera, un $61,56 \%$ piensan que sí, que el consentimiento es una forma de evitar responsabilidades por parte del profesional. Los pacientes más desconfiados fueron los que no tienen estudios o tan solo estudios primarios (tabla 3).

Anteriormente se mencionó que el $99,77 \%$ de los pacientes entendió claramente lo que se le haría en la operación y que ésta podía presentar complicaciones; sin embargo, ante la pregunta «ipodría enumerar alguna de las complicaciones explicadas? » un $35,32 \%$ no fue capaz de mencionar ni una sola complicación. Los pacientes universitarios son los que más complicaciones fueron capaces de repetir, no habiendo diferencias entre el sexo y el nivel de ansiedad (tabla 4). Las complicaciones más frecuentemente

\begin{tabular}{|c|c|c|}
\hline \multicolumn{3}{|c|}{$\begin{array}{r}\text { Tabla 3: Contestación a la pregunta i piensa u } \\
\text { consentimiento exime de responsabilidad al persor } \\
\text { según los parámetros estudiados }\end{array}$} \\
\hline & SI & No \\
\hline MUJ ERES & $138(58,97 \%)$ & $96(41,03 \%)$ \\
\hline HOMBRES & $99(65,56 \%)$ & $52(34,44 \%)$ \\
\hline SIN ESTUDIOS O PRIMARIOS & $51(85,00 \%)$ & $9(15,00 \%)$ \\
\hline ESTUDIOS SECUNDARIOS & $116(61,05 \%)$ & $74(38,95 \%)$ \\
\hline ESTUDIOS UNIVERSITARIOS & $70(51,85 \%)$ & $65(48,15 \%)$ \\
\hline ANSIEDAD BAJ A & $72(59,50 \%)$ & $49(40,50 \%)$ \\
\hline ANSIEDAD MEDIA & $94(62,67 \%)$ & $56(37,33 \%)$ \\
\hline ANSIEDAD ALTA & $71(62,28 \%)$ & $43(37,72 \%)$ \\
\hline ANSIEDAD MEDIA & $138(92,00 \%)$ & $12(8,00 \%)$ \\
\hline ANSIEDAD ALTA & $109(95,61 \%)$ & $5(4,39 \%)$ \\
\hline
\end{tabular}

\begin{tabular}{|c|c|c|}
\hline \multicolumn{3}{|c|}{$\begin{array}{l}\text { Tabla 4: Contestación a la pregunta ipodría enum } \\
\text { complicaciones explicadas? }\end{array}$} \\
\hline & SI & No \\
\hline MUJ ERES & $152(64,96 \%)$ & $82(35,04 \%)$ \\
\hline HOMBRES & $97(64,25 \%)$ & $54(35,76 \%)$ \\
\hline SIN ESTUDIOS O PRIMARIOS & $26(43,33 \%)$ & $34(56,67 \%)$ \\
\hline ESTUDIOS SECUNDARIOS & $113(59,47 \%)$ & $77(40,53 \%)$ \\
\hline ESTUDIOS UNIVERSITARIOS & $102(75,56 \%)$ & $33(24,44 \%)$ \\
\hline ANSIEDAD BAJ A & $84(69,42 \%)$ & $37(30,58 \%)$ \\
\hline ANSIEDAD MEDIA & $93(62,00 \%)$ & $57(38,00 \%)$ \\
\hline ANSIEDAD ALTA & $72(63,16 \%)$ & $42(36,84 \%)$ \\
\hline
\end{tabular}

enumeradas fueron la inflamación $(22,41 \%)$, afectación de nervios $(18,39 \%)$, dolor $(17,98 \%)$, daño en dientes vecinos $(7,82 \%$ y ya en menor proporción citan infección, alergia, hemorragia, hematoma, sinusitis, fractura ósea, rotura de instrumentos, etc. (fig. 3).

Quisimos saber si a los pacientes les parecía útil este tipo de informa- ción y un 98,96\% contestaron que les parecía útil (tabla 5).

Las dos últimas preguntas hacían referencia a si este tipo de información aumentaba en los pacientes el nivel de ansiedad o por el contrario les tranquilizaba. La mayoría de los pacientes $(68,31 \%)$ afirmaba que el conocer las posibles complicaciones no aumenta en ellos el nivel de ansiedad y a un 


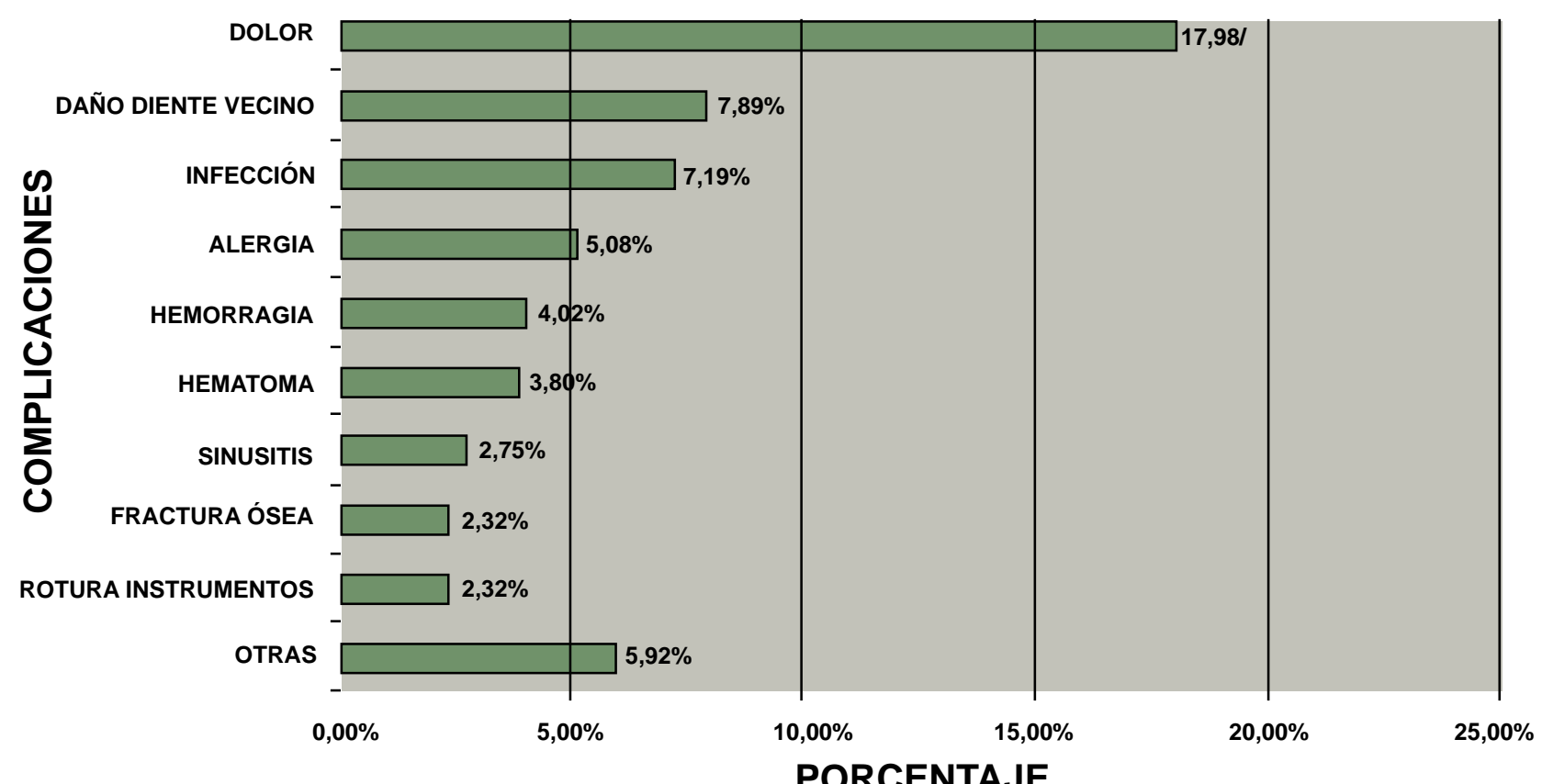

Figura 3. Tipo de complicación que el paciente es capaz de recordar de las que figuraban en el consentimiento.

$46,23 \%$ le tranquiliza que le den este tipo de información (tablas 6 y 7).

\section{Discusión}

La Ley General de Sanidad ${ }^{8}$ reconoce el derecho del paciente a que «se le dé en términos comprensibles, a él y a sus familiares o allegados, información completa y continuada, verbal y escrita, sobre su proceso, incluyendo diagnóstico, pronóstico y alternativas de tratamiento»... "«siendo preciso el previo consentimiento escrito del usuario para la realización de cualquier intervención...»

A pesar de la claridad de esta ley todavía son muchos los profesionales que intervienen a sus pacientes sin el consentimiento informado, tanto en Odontología como en otras ramas de la Medicina. Guix Oliver ${ }^{6 * *}$ examinó los registros clínicos de 314 pacientes de

\begin{tabular}{|lcc|}
\hline \multicolumn{3}{|c|}{ Tabla 5: Respuesta acerca de si les parece útil este tipo de } \\
información
\end{tabular}

cirugía y sólo el 78\%contenía formularios de consentimiento informado; el diagnóstico estaba presente en el $14,9 \%$ de estos formularios y menos de la mitad de ellos había sido firmado por el médico que dio la información, lo cual es obligatorio. Esto indica, según el autor, que los médicos no están totalmente involucrados en el proceso del consentimiento informado.

Los profesionales temen que el paciente se asuste con la información suministrada. Sin embargo, según los resultados de nuestro estudio ningún paciente rechazó el tratamiento ante la explicación de las posibles complicacio- 
nes a pesar de que prácticamente todos sabían que podían negarse a la intervención. En el presente estudio los pacientes venían derivados de otros centros (especialmente de la Seguridad Social) específicamente para la extracción quirúrgica de sus terceros molares; ello puede explicar que ninguno se negara a la intervención al conocer las posibles complicaciones. Sería necesario hacer un estudio similar con pacientes privados de una consulta de odontología general a los que se les recomienda la extracción de los cordales, pues quizás estos pacientes no sienten tanto la necesidad de la extracción. Queda la duda sobre si en este otro grupo se producirían negativas.

El consentimiento debe hacerse para que cada paciente pueda comprender completamente los datos suministrados de modo que esté capacitado para tomar una decisión verdaderamente informada. En nuestra encuesta el 99,77\% afirma haber entendido lo que se le haría en la operación y que ésta podía presentar complicaciones, sin embargo el $35,32 \%$ no fue capaz de mencionar ni una sola complicación, lo que bajaría el nivel de comprensión hasta un $64,45 \%$ En un estudio similar realizado por Parera de Cáceres $^{9 * *}$, sobre endoscopia gastrointestinal, el nivel de comprensión se cifró en un 90,7\% Esto estuvo relacionado con el nivel de educación, pues los universitarios fueron los que más complicaciones eran capaces de repetir. Lo mismo ocurrió en el estudio de Guix Oliver ${ }^{6 * *}$ sobre pacientes sometidos a intervenciones quirúrgicas en dos hospitales de Cataluña donde hubo menor comprensión por parte de los pacientes con menor nivel sociocultural.

\begin{tabular}{|c|c|c|}
\hline \multicolumn{3}{|c|}{$\begin{array}{r}\text { Tabla 6: Sobre si el conocer las posibles complicaci } \\
\text { el paciente el nivel de ansiedad }\end{array}$} \\
\hline & SI & No \\
\hline MUJ ERES & $84(35,89 \%)$ & $150(64,11 \%)$ \\
\hline HOMBRES & $38(25,17 \%)$ & $113(74,83 \%)$ \\
\hline SIN ESTUDIOS O PRIMARIOS & $13(21,67 \%)$ & $47(78,33 \%)$ \\
\hline ESTUDIOS SECUNDARIOS & $61(32,11 \%)$ & $129(67,89 \%)$ \\
\hline ESTUDIOS UNIVERSITARIOS & $48(35,56 \%)$ & $89(64,44 \%)$ \\
\hline ANSIEDAD BAJ A & $36(24,79 \%)$ & $91(75,21 \%)$ \\
\hline ANSIEDAD MEDIA & $40(26,67 \%)$ & $110(73,33 \%)$ \\
\hline ANSIEDAD ALTA & $52(45,61 \%)$ & $62(54,39 \%)$ \\
\hline
\end{tabular}

\begin{tabular}{|lcr|}
\hline \multicolumn{3}{|c|}{$\begin{array}{c}\text { Tabla 7: Respuesta de los pacientes sobre si el conocer las posibles } \\
\text { complicaciones les tranquiliza }\end{array}$} \\
\hline MUJ ERES & SI \\
\hline HOMBRES & $99(42,31 \%)$ & $135(57,69 \%)$ \\
\hline SIN ESTUDIOS O PRIMARIOS & $79(52,32 \%)$ & $72(47,68 \%)$ \\
\hline ESTUDIOS SECUNDARIOS & $32(53,33 \%)$ & $28(46,67 \%)$ \\
\hline ESTUDIOS UNIVERSITARIOS & $91(47,89 \%)$ & $99(52,11 \%)$ \\
\hline ANSIEDAD BAI A & $55(40,74 \%)$ & $80(59,26 \%)$ \\
\hline ANSIEDAD MEDIA & $65(53,72 \%)$ & $56(46,28 \%)$ \\
\hline ANSIEDAD ALTA & $75(50,00 \%)$ & $75(50,00 \%)$ \\
\hline
\end{tabular}

Los profesionales pueden hacer un uso positivo del consentimiento informado para ganar la confianza de los pacientes, pues éstos comprueban que se cuenta con ellos en su tratamiento. De hecho, en nuestra encuesta a un 98,96\%les parece útil este tipo de información. La mayoría de los pacientes no se pone más nervioso por conocer las complicaciones e incluso a casi la mitad de ellos les tranquiliza que les expliquen lo que se les va a hacer y lo que puede ocurrir. Esto indica que el consentimiento debe ser algo más que la simple firma de un impreso $^{6 * *}$. Debe ser un intercambio de información, comprensión, confianza y consentimiento entre profesional y paciente.

L que nos pareció más negativo de las respuestas de los pacientes es que gran parte de éstos crean que el consentimiento sirve para eximir de responsabilidad al profesional. En nuestro caso el porcentaje es mayor que el observado por Parera de Cáceres $^{9 * *}$ : el $42 \%$ de sus pacientes opinaba que el propósito del consentimiento 
es eximir al doctor de responsabilidad. El profesional que informa debe saber transmitir al paciente que esto no es así, que lo único que se pretende es respetar sus derechos.

Por tanto debemos tener presente que la extracción quirúrgica del tercer molar inferior es una maniobra no exenta de riesgos y por ello es imprescindible un consentimiento informado, para que el paciente comprenda las posibles complicaciones, las alternativas terapéuticas y acepte los riesgos de la intervención. Según Perea Pére $z^{10^{* *}}$, los riesgos que no asume el paciente los estamos asumiendo nosotros. No debemos realizar ningún tratamiento de Cirugía Bucal que no venga precedido de una correcta y completa historia clínica, un consentimiento informado ${ }^{11}$ y las pruebas complementarias adecuadas al caso.

\section{Conclusiones}

El consentimiento informado fue comprendido por la mayoría de los pacientes y ninguno de ellos se negó a la intervención al conocer las posibles complicaciones. Lama la atención que muchos pacientes creen que el consentimiento sirve para eximir de responsabilidad al dentista.

\section{Bibliografía recomendada}

Para profundizar en la lectura de este tema, el/los autor/es considera/an interesantes los artículos que aparecen señalados del siguiente modo: *de interés $* *$ de especial interés.

$1 * *$. Ursu SC. Legal considerations in clinical decisión making. J Dent Educ 1992;56(12): 808-11.

El aumento de las denuncias en odontología influye en la toma de decisiones en los tratamientos.

2**. Zarandieta G. Estudio de las reclamaciones odontológicos-legales en la Ciudad de Madrid. Años 1990-1994. Tesina de Licenciatura. Universidad Complutense de Madrid. Facultad de Odontología. Madrid, 1996.

El autor hace un estudio muy exhaustivo sobre las denuncias que se han producido en la ciudad de Madrid; analiza qué ramas de la Odontología reciben más demandas y cuáles pueden ser las causas de éstas.

3. Morris WO. The damndest things have occurred in the other fellow's dental offices. J Law Ethics Dent 1991;4:79-88.

4. Donado Rodríguez M. Cirugía Bucal. Patología y Técnica. $1^{\text {a }}$ edición. Madri: Ed. el autor, 1990. $5^{* *}$. Conrad SM, Blakey GH, Shugars DA, Marcia- ni RD, Phillips C, White RP. Patients'perception of recovery after third molar surgery. $\mathrm{J}$ Oral Maxillofac Surg 1999;57(11):1288-94. La percepción que el paciente tiene de su postoperatorio es, a menudo, distinta de la del profesional, por lo que se le debe dar información detallada sobre la intervención y el postoperatorio.

6**. Guix Oliver J, Balana L, Carbonel JM, y cols. The perfomance and perception of informed consent in a health-care section of Catalonia. Rev Esp Salud Publica 1999; 73(6):669-75.

El consentimiento informado no se estaba realizando correctamente en dos hospitales analizados, pues sólo el $78 \%$ de las historias clínicas lo contenían y menos del $50 \%$ estaba firmado por el médico.

7**. Cannavina CD, Cannavina G, Walsh TF. Effects of evidence-based treatment and consent on profesional autonomy. $\mathrm{Br}$ Dent $\mathrm{J}$ 2000;188(6):302-6.

Los tratamientos en Odontología, al igual que en cualquier otra rama de la Medicina, deben estar basados en la evidencia. Se debe implicar al paciente en el tratamiento al que se va a someter.

8. Ley 25 abril 1986, núm 17/86 (Jefatura del estado).SANIDAD. Ley General de Sanidad. B.O.E. 28 y 29 ABRIL, 1986 (NUMS.101 y 102).

9**. Parera de Cáceres A, González-Asanza C, Menchen PL, et al. Survey of informed consent in a gastrointestinal endoscopy unit. Gastroenterol Hepatol 2000;23(7):317-21.

Los autores realizaron una encuesta entre los pacientes sometidos a endoscopia gastrointestinal para conocer si comprendieron el consentimiento informado previo.

10**. Perea Pérez B, Vega del Barrio JM. Medidas para prevenir la demanda o limitar sus consecuencias. Prof Dent 2001;4(1):17-21.

Los autores dan una serie de recomendaciones para evitar las demandas.

11. Dayal L, Cannel H. Informed consent and the practice of good dentistry. $\mathrm{Br}$ Dent $\mathrm{J}$ 1995; 178:454-60 